\title{
A plasma-assisted bioextrusion system for tissue engineering
}

DOI:

10.1016/j.cirp.2018.04.077

Document Version

Accepted author manuscript

Link to publication record in Manchester Research Explorer

\section{Citation for published version (APA):}

Liu, F., Wang, W., Mirihanage, W., Hinduja, S., \& Da Silva Bartolo, P. J. (2018). A plasma-assisted bioextrusion system for tissue engineering. CIRP Annals - Manufacturing Technology. https://doi.org/10.1016/j.cirp.2018.04.077

\section{Published in:}

CIRP Annals - Manufacturing Technology

\section{Citing this paper}

Please note that where the full-text provided on Manchester Research Explorer is the Author Accepted Manuscript or Proof version this may differ from the final Published version. If citing, it is advised that you check and use the publisher's definitive version.

\section{General rights}

Copyright and moral rights for the publications made accessible in the Research Explorer are retained by the authors and/or other copyright owners and it is a condition of accessing publications that users recognise and abide by the legal requirements associated with these rights.

\section{Takedown policy}

If you believe that this document breaches copyright please refer to the University of Manchester's Takedown Procedures [http://man.ac.uk/04Y6Bo] or contact uml.scholarlycommunications@manchester.ac.uk providing relevant details, so we can investigate your claim.

\section{OPEN ACCESS}


Annals - Manufacturing Technology

Elsevier Editorial system(tm) for CIRP

Manuscript Draft

Manuscript Number: 2018-E-30R2

Title: A plasma-assisted bio-extrusion system for tissue engineering

Article Type: STC E

Keywords: Additive manufacturing, biomedical, tissue engineering

Corresponding Author: Professor srichand hinduja, B.E., MSC, PhD, FCIRP, FIMechE

Corresponding Author's Institution: University of Manchester

First Author: F Liu

Order of Authors: F Liu; W Wang; W Mirihanage; srichand hinduja, B.E., MSc, PhD, FCIRP, FIMechE; Paulo J Bartolo

Abstract: A challenge for tissue engineering is to produce synthetic scaffolds of adequate chemical, physical and biological cues effectively. This paper describes a plasma-assisted bio-extrusion system to produce functional-gradient scaffolds; it comprises pressure-assisted and screwassisted extruders, and plasma jets. This paper also describes how the system conducts plasma surface modification during the polycaprolactone scaffold fabrication process. Water contact angle and in vitro biological tests confirm that the plasma modification alters the hydrophilicity properties of synthetic polymers and promotes proliferation of cells, leading to homogeneous cell colonization. The results suggest this system is promising for producing functional gradient scaffolds of biomaterials. 


\title{
A plasma-assisted bioextrusion system for tissue engineering
}

\author{
F. Liu, W. Wang, W. Mirihanage, S. Hinduja (1) and P. J. Bartolo (1) \\ School of Mechanical, Aerospace and Civil Engineering, The University of Manchester, UK
}

A challenge for tissue engineering is to produce synthetic scaffolds of adequate chemical, physical and biological cues effectively. This paper describes a plasma-assisted bio-extrusion system to produce functional-gradient scaffolds; it comprises pressure-assisted and screw-assisted extruders, and plasma jets. This paper also describes how the system conducts plasma surface modification during the polycaprolactone scaffold fabrication process. Water contact angle and in vitro biological tests confirm that the plasma modification alters the hydrophilicity properties of synthetic polymers and promotes proliferation of cells, leading to homogeneous cell colonization. The results suggest this system is promising for producing functional gradient scaffolds of biomaterials.

\section{Additive manufacturing, biomedical, tissue engineering}

\section{Introduction}

Tissue engineering is promising for organ replacement as it minimizes the side effects of organ transplantation. Biomanufacturing is one of the key stages in tissue engineering as it is concerned with the development of biological substitutes that restore, maintain, or improve tissue function. Biomanufacturing preferably requires the combined use of additive manufacturing (AM), biocompatible and biodegradable materials, cells and biomolecular signals [1]. AM techniques are commonly applied in scaffold fabrication due to their superior ability in controlling pore size, shape and distribution, thus creating interconnected porous structures [2,3]. However, cell-seeding and proliferation efficiency are currently big challenges due to the following limitations [4-6].

(i) Most AM techniques are limited to single-material fabrication, which makes it difficult to provide an appropriate environment for cells due to inadequate chemical, physical and biological cues provided during the AM process.

(ii) Non-uniform cell distribution, with rare cell adhesion in the core region of scaffolds, often caused by tortuosity of the constructs.

(iii) Limited cell colonization due to the hydrophobicity of the most commonly used synthetic biopolymers.

Different strategies have been explored to overcome the above limitations. Different materials have been developed and utilized to produce multiple-material scaffolds [7-9]. However, most of these systems can only fabricate the scaffolds from one type of biomaterial, either soft hydrogels containing cells or bio-signals (the average Young's modulus is a few $\mathrm{kPa}$ ), or rigid biopolymers and composites (the average Young's modulus is a few hundred $\mathrm{MPa}$ ). The disadvantage with the latter is that it fails to mimic natural tissues. Increasing the hydrophilicity of polymer scaffold to achieve a better cell attachment rate can be achieved by different chemical surface treatment methods. One of them uses a simple sodium hydroxide treatment, rendering the scaffolds more hydrophilic [10]. A low-temperature plasma modification is also capable of improving the hydrophilicity of biopolymers by inducing certain functional groups on the surface to change the chemical signalling, wettability and energy without altering the bulk properties [11-14]. These researchers conducted the plasma treatment after the scaffolds were printed, thus limiting the penetration depth, which subsequently resulted in a non-uniform cell distribution along the scaffold.

This paper introduces a novel plasma-assisted bioextrusion system (PABS), which represents an advancement in the manufacture of functional-gradient scaffolds, as it not only permits the processing of different biomaterials but also makes in-process plasma surface modification possible. The system can print a structure and deposit amine groups layer by layer within the scaffold with Nitrogen-based plasma modification.

The water contact angle (WCA) is used to evaluate the hydrophilicity changes in polycaprolactone (PCL) scaffolds after a $\mathrm{N}_{2}$ plasma treatment. In vitro biological assessment with Human Adipose Derived Stem Cells (hADSC) has been performed to evaluate the adhesion and proliferation on treated and untreated scaffolds.

\section{Materials and Methods}

\subsection{Materials}

PCL (CAPA®6500, Mw $=50,000 \mathrm{~g} / \mathrm{mol}$ ) is a semi-crystalline polymer with a density of $1.1 \mathrm{~g} / \mathrm{cm}^{3}$, a melting temperature between $58-60{ }^{\circ} \mathrm{C}$ and a glass transition temperature of $-60{ }^{\circ} \mathrm{C}$. This polymer was purchased, in the form of $3 \mathrm{~mm}$ pellets, from Perstorp (Cheshire, United Kingdom) and used in this project.

\subsection{Experimental Rig}

The experimental rig (Fig. 1(a)) described in $[15,16]$ has been further developed to include the plasma modification unit which is described in detail below. For the sake of completeness, some of the more important parts of the rig are also briefly mentioned below.

(i) The rig has of a multi-extrusion unit consisting of three heads, two pressure-assisted and one screw-assisted. The extrusion unit has four movements, one rotational movement (C1) for indexing the extrusion heads, a second rotational movement for controlling the screw rotational speed of the screw-assisted extruder (C2) and two movements ( $\mathrm{X}$ and $\mathrm{Y}$ axes) in the $\mathrm{X}-\mathrm{Y}$ plane; all four movements are controlled by CNC drives. 


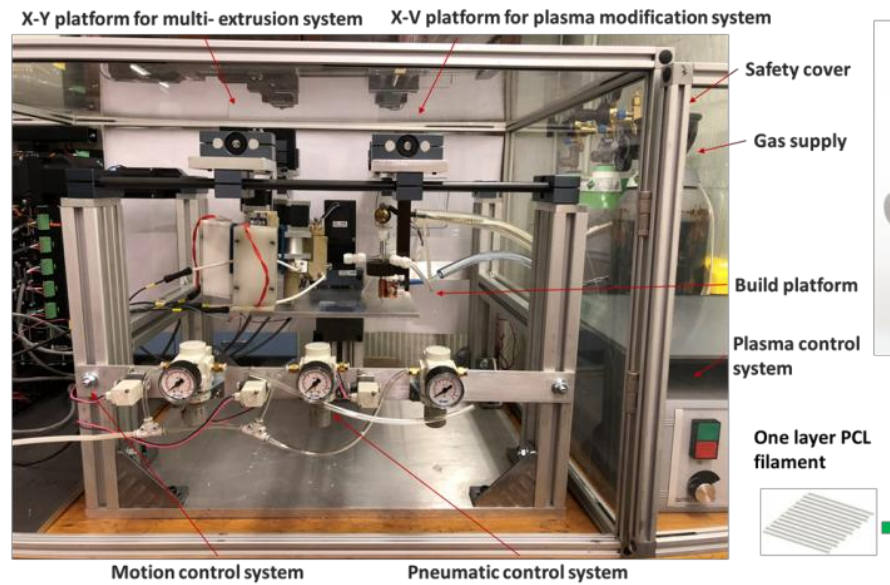

(a)

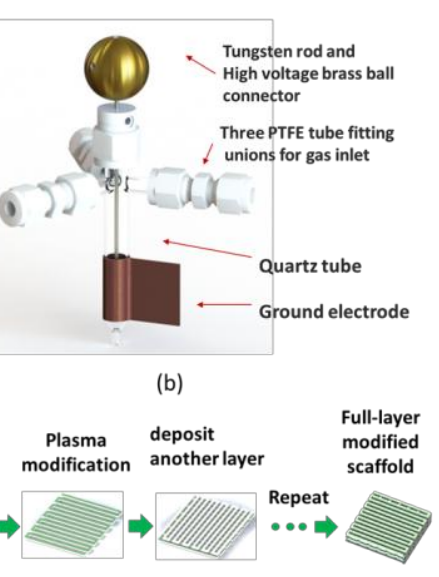

(c)

Fig. 1 (a) PABS consisting of a control system, multi-extrusion heads, and plasma modification unit; (b) plasma modification unit comprising a glass tube reaction jet with three gas inlets for gas mixture and different chemical groups deposition; (c) sequence of operations to fabricate full-layer plasma treated scaffolds.

(ii) The plasma unit is an atmospheric plasma jet and is mounted on an X-Y platform, which is co-planar with the extrusion platform. Since both the platforms share common cylindrical guide rails in the $X$-direction, the movement of this platform in the $\mathrm{X}-\mathrm{Y}$ plane gives rise to only one additional axis ( $\mathrm{V}$ axis), the fifth axis of the rig. A quartz capillary (outside diameter $=7$ $\mathrm{mm}$; inner diameter $=5 \mathrm{~mm}$; length $=70 \mathrm{~mm}$ ) with three gas inlets serves as the reaction jet. A tungsten $\operatorname{rod}(\mathrm{D}=2 \mathrm{~mm})$ and one copper film (width $=10 \mathrm{~mm}$ ) wrapped around the quartz tube serve as the high-voltage and ground electrodes, respectively. The electrode is connected to a high-voltage DC power supply (applied voltage $=10 \mathrm{kV}$; frequency $=50 \mathrm{kHz}$ ). The plasma is generated from the top central electrode, expanding to the surrounding air inside and outside the nozzle (Fig. 1(b)).

(iii) The build platform moves in the z-direction and constitutes the sixth controllable axis.

Two different types of extrusion heads have been incorporated in the rig, so that scaffolds can be fabricated from materials with both low and high viscous materials such as hydrogels, biomaterials and polymer composites. The stepper motors rotate the extrusion unit, control the rotational speed of the extruder screw, and drive the two platforms in the X-Y plane. Because they are CNC controlled, it means that the region to be plasma treated in a layer of the scaffold, can be of any arbitrary shape; this region is defined using a user-friendly interface, and then the in-house software automatically generates a word-address program, containing G, M, etc. codes, to move the plasma modification unit over the user-defined region. In the work reported herein, for the sake of simplicity, the entire area of each layer is plasma modified. Figure 1(c) shows the steps to fabricate full-layer modified scaffolds; the plasma modification is carried out after each layer of the scaffold has been printed, thereby sandwiching plasma-induced chemical membranes between printed layers of the scaffold.

Scaffolds with a cross-section of $10 \times 10 \mathrm{~mm}$ and a height of $3 \mathrm{~mm}$ were fabricated using the single lay pattern of $0 / 90^{\circ}$ and a filament distance of one $\mathrm{mm}$ for fabricating inter-connected structures. The plasma treatment was conducted at a pressure of $0.689 \mathrm{bar}$ and a flow rate of $5 \mathrm{l} / \mathrm{mm}$. The deposition speed of the plasma jet was $3 \mathrm{~mm} / \mathrm{s}$ and each layer was subjected to the plasma treatment for one minute. The distance from the bottom of the jet to the surface of the PCL filaments was $10 \mathrm{~mm}$. The other processing conditions for the scaffolds were: slice thickness
$=0.5 \mathrm{~mm}$; liquefier temperature $=90^{\circ} \mathrm{C}$; extrusion screw rotational speed $=15 \mathrm{rpm}$; and nozzle tip size $=0.5 \mathrm{~mm}$. This rotational speed is higher than that used in [15] because it was found that higher speeds, the mechanical behaviour of the scaffold is improved [17].

\subsection{Morphological characterization}

Scanning electron microscopy (SEM) was used to image the morphology of the scaffolds for visualization and evaluation of the surface characteristics for both plasma-treated and untreated scaffolds. The scaffolds were coated with platinum $(\sim 40 \mathrm{~s}$ sputtering) and imaged at $10 \mathrm{kV}$ (Hitachi S3000N). These images were then analysed using ImageJ [18] to measure the fibre diameter and pore size, and to ascertain whether the plasma surface modification process has a physical impact on these geometrical parameters. At least 40 measurements were made each time.

\subsection{Wettability measurement}

Water Contact Angle (WCA) tests on the surfaces of untreated and plasma-treated PCL scaffolds were carried out with a commercial KSV CAM 200 system (KSV Instruments, Finland). The system is equipped with a CCD video camera and a micrometric liquid dispenser to drop $2 \mu l$ of distilled water on the surface of the scaffold. The $\mu l$ measurements of the contact angles are automatically calculated with the instrument software. The temporal effect of plasma treated scaffolds was also assessed with WCA tests on days 1, 3, 7 and 14 .

\subsection{Mechanical compression test}

Compression tests were conducted to determine the effect of full-layer plasma modification on the mechanical behaviour of the scaffolds. Each test was repeated four times at a speed of 0.5 $\mathrm{mm} / \mathrm{min}$ and a maximum strain value of 0.45 , using an INSTRON 3344 testing device with a $2000 \mathrm{~N}$ load cell.

\subsection{Biological test}

In vitro biological assessments were conducted with human adipose-derived stem cells (STEMPRO, Invitrogen, Waltham, MA, USA). Before cell seeding, the scaffolds were sterilized by soaking in $70 \%$ ethanol for 2 hours. After sterilisation, samples were rinsed twice in phosphate buffered saline (Gibco, ThermoFisher Scientific, Waltham, MA, USA), transferred to 24-well plates and air-dried for 24 hours at room temperature. 50,000 cells were 
seeded on each sample, including plasma-treated and untreated scaffolds.

Cell viability/proliferation behaviour and the percentage of cells attached to the scaffolds (cell-seeding efficiency) were assessed through Alamar Blue assay (also referred to as Resazurin assay, reagents from Sigma-Aldrich, UK) [19]. Cell viability/proliferation was measured on the first, third, seventh and fourteenth day after cell seeding [20]. For each measurement, cell-seeded scaffolds were transferred to a new 24-well plate and $0.7 \mathrm{~mL}$ of Alamar Blue solution was added to each well, the plate was incubated for four hours under standard conditions $\left(37^{\circ} \mathrm{C}\right.$, $5 \% \mathrm{CO}_{2}$ and $95 \%$ humidity). After incubation, $150 \mu \mathrm{l}$ of each sample solution was transferred to a 96-well plate and the fluorescence intensity measured at $540 \mathrm{~nm}$ excitation wavelength and $590 \mathrm{~nm}$ emission wavelength with a spectrophotometer (Sunrise, Tecan, Männedorf, Zurich, Switzerland) [21]. Each test was repeated thrice.

\subsection{Data analysis}

All data are represented herein as mean \pm standard deviation. Biological results were subjected to one-way analysis of variance (one-way ANOVA) and post hoc Tukey's test using GraphPad Prism software. Significance levels were set at $p<0.05$.

\section{Results and Discussion}

\subsection{Morphology properties}

SEM images of the 3D structures are shown in Fig. 2(a). SEM images of the top and side views clearly show a well-bonded structure; although the structure was programmed to have a uniform pore size of $500 \times 500 \mu \mathrm{m}$, this was not the case in practice. For instance, in the left image in Fig. 2(a), the four pores are not of the same size, and their dimensions vary between $386 \pm 15 \mu \mathrm{m}$ to $567 \pm 7 \mu \mathrm{m}$; this variation in the pore size can probably be attributed mostly to the positioning errors in the open-loop stepper motor drives.

Figure 2(b) shows SEM images of filament surfaces of top layers from untreated and plasma-treated scaffolds. The results confirm that the plasma surface modification has an impact on the surface roughness, which is an important factor in biomedical materials, as it may affect cell adhesion [19]. The surface of an untreated filament has small pores $(\sim 5-20 \mu \mathrm{m}$ diameters $)$. After treatment, these pores disappear; instead lines in the direction of the plasma jet movement are generated, which result in a rougher surface topography. Their occurrence is due to the gas flow generated by the plasma jet during the modification process. Because the plasma modification is done during the fabrication process, it means that the material is still in the molten state when the modification is performed, resulting in the gas flow having a more pronounced effect on the surface topography.

\subsection{Surface wettability characterization}

Since synthetic polymers, such as PCL, are hydrophobic, it is important to know the surface wettability before and after the plasma treatment. WCA measurements were performed on untreated and fully treated plasma PCL scaffold surfaces to determine the effect of plasma modification on the surface wettability. Table 1 highlights the WCA results at different instances after the droplet was dropped on the surface of the scaffolds. The results show that in the case of an untreated PCL scaffold, there is little or no change in the WCA values with time. These values vary between $83.2 \pm 2.0^{\circ}$ and $80.9 \pm 2.7^{\circ}$. For the treated scaffolds, the WCA value, at $0 \mathrm{~s}$, was lower at $63.0 \pm 3.1^{\circ}$, leading to a fully wetting value of $26.7 \pm 0.9^{\circ}$ at $0.5 \mathrm{~s}$. The droplet (a)
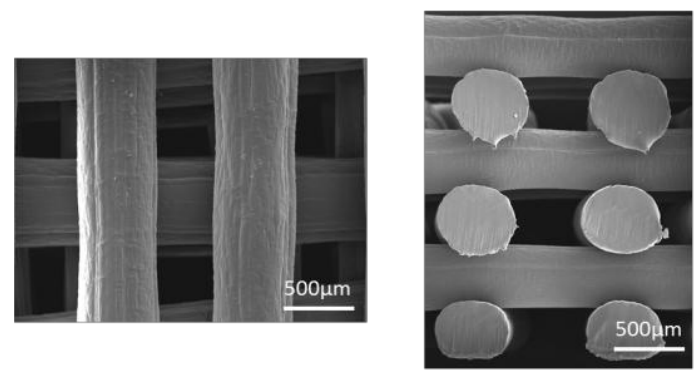

(b)
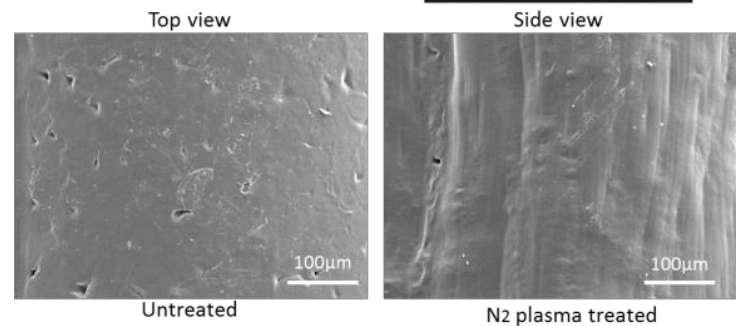

Figure 2. SEM images of (a) top and cross-section views of 3D printed PCL scaffolds after $\mathrm{N}_{2}$ plasma treatment; (b) top view of filament surfaces of untreated and plasma-treated scaffolds.

was quickly absorbed into the structure and no value was obtained at $3 \mathrm{~s}$. The results reveal that the hydrophilicity of the surface is dramatically improved due to the ionisable groups introduced on the surface by the $\mathrm{N}_{2}$ plasma, which enhances the hydrogen bonding with water. When these results are compared with those published in $[11,14]$, the absorption speed is faster, probably because, with each layer being plasma modified, chemical heterogeneity is generated on the surface of every layer.

Figure 3 shows the temporal variation of WCA but on a much longer scale; the WCA values increase non-linearly with time, which means the plasma modification effect decreases with time. These changes can be attributed to the structural rearrangement of polymer chains to decrease surface energy [22]. These results confirm that PABS has the capability of creating highly hydrophilic structures but the effect decreases with time.

\subsection{Mechanical properties characterization}

The stress-strain curves (Fig.4) for both untreated and fullytreated PCL scaffolds reveal that the mechanical behaviour of both scaffolds is almost identical. Except for some non-linearity between strains of 0.1 and 0.25 , the stress-strain curves exhibit a linear variation. These observations indicate that the in-process plasma modification process does not affect the mechanical properties of scaffolds.

\subsection{Biological characterization}

The adhesion and proliferation of hADSCs on plasma full-layer modified PCL scaffolds was studied and compared with untreated ones. The biological characterization was assessed using Alamar Blue Assay. The fluorescence intensity of cells seeded scaffolds was measured at four different culture time points $(1,3,7$ and 14 days), as shown in Fig. 5. Higher fluorescence intensity corresponds to more metabolically active cells. Comparing the results at the four different time points, it can be observed that for both plasma-treated and untreated scaffolds, the fluorescence intensity, which represents the growth in cell viability/proliferation, increases with time, suggesting that scaffolds fabricated with this particular additive manufacturing system are suitable substrates for cell proliferation. Comparing the fluorescence intensities on day one (see Fig. 4), there is no significant difference between the plasma treated and untreated scaffolds. But on day three, the plasma treated scaffolds present a statistically higher fluorescence intensity than the non-treated scaffolds, suggesting a higher cell attachment/proliferation rate 
Table 1 Temporal variation of water contact angles for treated and untreated scaffolds

\begin{tabular}{lll} 
& PCL scaffolds & $\mathrm{N}_{2}$ plasma fully treated \\
\hline $0 \mathrm{~s}$ & $83.2 \pm 2.0$ & $63.0 \pm 3.1$ \\
\hline $0.5 \mathrm{~s}$ & $82.9 \pm 1.2$ & $26.7 \pm 0.9$ \\
\hline $3 \mathrm{~s}$ & $80.9 \pm 2.7$ & Fully absorbed
\end{tabular}

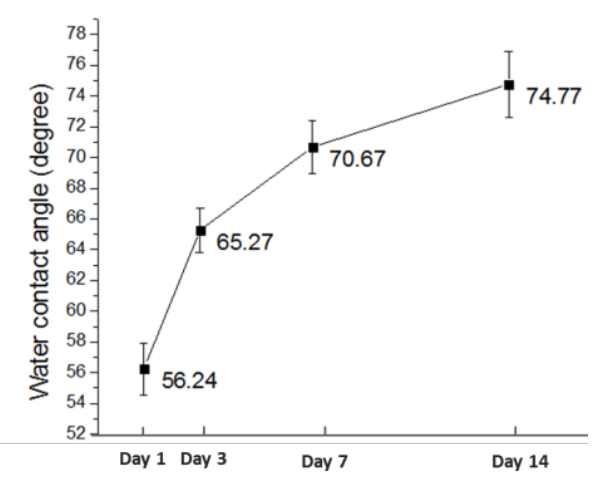

Fig. 3 Temporal variation of the water contact angle for treated scaffolds

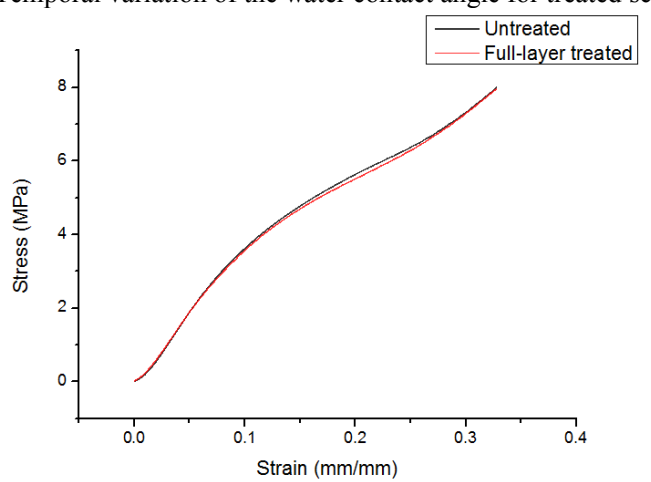

Fig. 4 Typical stress-strain curve for untreated and full-layer $\mathrm{N}_{2}$ plasma treated PCL scaffolds.

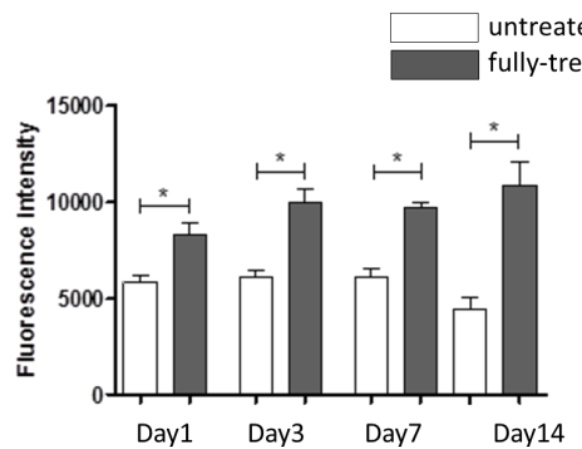

Fig. 5 Temporal variation of fluorescence intensity of cell-seeded PCL scaffolds with and without $\mathrm{N}_{2}$ plasma treatment.

caused by the plasma surface modification through increased hydrophilicity. The phenomenon also exists on the seventh and fourteenth day.

\section{Conclusions and outlook}

This paper describes the development of a novel additive manufacturing system comprising a multi-extrusion system and a plasma jet unit, enabling in-process plasma modification during scaffold printing to create a full-layer modified structure. The effects of full-layer plasma treatment on PCL scaffold were examined and exhibit the following advantages:

(i) SEM analysis show the capability of PABS to produce full-layer plasma treated scaffolds with fully interconnected channels and regular pore size. (ii) The surface morphology of filament is affected due to the plasma jetting.

(iii) WCA results confirm that the hydrophilic character of the PCL samples increased due to the amide groups introduced by plasma jetting.

(iv) As confirmed from the mechanical analyses, the inprocess modification process does not change the compressive mechanical performance of the scaffolds.

(v) The treated scaffolds exhibit a higher cell attachment/proliferation rate suggesting an improved biological performance.

Applications that may benefit from this technology include hybrid tissue, which has compositional variations depending on the region or organ-like structure which require continuous vascular network to facilitate nutrient diffusion.

\section{References}

[1] Bartolo, P., Kruth, J.P., Silva, J., Levy, G., Malshe, A., Rajurkar, K., Mitsuishi, M., Ciurana, J., Leu, M., 2012. Biomedical production of implants by additive electrochemical and physical processes. CIRP Annals-Manufacturing Technology, 61/2, 635-655.

[2] Khani, N., Nadernezhad, A., Bartolo, P., Koc, B., 2017. Hierarchical and spatial modeling and bio-additive manufacturing of multi-material constructs. CIRP Annals, $66 / 1,229-232$.

[3] Vyas, C., Pereira, R., Huang, B., Liu, F., Wang, W., Bartolo, P., 2017, Engineering the vasculature with additive manufacturing, Current Opinion in Biomedical Engineering, 2: 1-13.

[4] Giannitelli, S.-M., Mozetic, P., Trombetta, M., Rainer, A., 2015, Combined additive manufacturing approaches in tissue engineering, Acta biomaterialia, 24: 1-11.

[5] Sobral, J.-M., Caridade, S.-G., Sousa, R.-A., Mano, J.-F., Reis, R.-L., 2011, Threedimensional plotted scaffolds with controlled pore size gradients: effect of scaffold geometry on mechanical performance and cell seeding efficiency, Acta Biomaterialia, 7/3: 1009-1018.

[6] Oh, S.-H., Lee, J.-H., 2013, Hydrophilization of synthetic biodegradable polymer scaffolds for improved cell/tissue compatibility, Biomedical materials, 8/1: 014101.

[7] Ozbolat, I.-T., Chen, H., Yu, Y., 2014, Development of 'Multi-arm Bioprinter' for hybrid biofabrication of tissue engineering constructs, Robotics and ComputerIntegrated Manufacturing, 30/3: 295-304.

[8] Murphy, S.-V., Atala, A., 2014, 3D bioprinting of tissues and organs, Nature biotechnology, 32/8: 773-785.

[9] Rutz, A. L., Hyland, K. E., Jakus, A. E., Burghardt, W. R., Shah, R. N., 2015. A multimaterial bioink method for 3D printing tunable, cell-compatible hydrogels. Advanced Materials, 27/9, 1607-1614.

[10] Wang, W., Caetano, G., Ambler, W.-S., Blaker, J.-J., Frade, M.A., Mandal, P., Diver, C., Bartolo, P., 2016, Enhancing the hydrophilicity and cell attachment of 3D printed PCL/Graphene scaffolds for bone tissue engineering, Materials, 9/12:992.

[11] Intranuovo, F., Gristina, R., Brun, F., Mohammadi, S., Ceccone, G., Sardella, E., Favia, P., 2014, Plasma Modification of PCL Porous Scaffolds Fabricated by Solvent-Casting/Particulate-Leaching for Tissue Engineering, Plasma Processes and Polymers, 11/2: 184-195.

[12] Hasan, A., Memic, A., Annabi, N., Hossain, M., Paul, A., Dokmeci, M. R., Khademhosseini, A., 2014, Electrospun scaffolds for tissue engineering of vascular grafts, Acta biomaterialia, 10/1: 11-25.

[13] Ko, Y. M., Choi, D.-Y., Jung, S.-C., Kim, B.-H., 2015, Characteristics of plasma treated electrospun polycaprolactone (PCL) nanofiber scaffold for bone tissue engineering, Journal of nanoscience and nanotechnology, 15/1: 192-195

[14] Intranuovo, F., Gristina, R., Fracassi, L., Lacitignola, L., Crovace, A., Favia, P., 2016, Plasma processing of scaffolds for tissue engineering and regenerative medicine, Plasma Chemistry and Plasma Processing, 36/1: 269-280.

[15] Liu, F., Hinduja, S., Bartolo, P., 2017, Design, fabrication and initial evaluation of a novel hybrid system for tissue engineering applications, Procedia CIRP, 65: 213218.

[16] Liu, F., Hinduja, S., Bartolo, P., 2018, User interface tool for a novel plasmaassisted bio-additive extrusion system, Rapid Prototyping Journal, DOI: 10.1108/RPJ-07-2016-0115.

[17] Liu, F., Vyas, C., Poologasundarampillai, G., Pape, I., Hinduja, S., Mirihanage, W. Bartolo, P. Structural evolution of PCL during melt extrusion 3D printing, Macromolecular Materials and Engneering, 303/2: 1700494.

[18] Schneider, C.-A., Rasband, W.-S., Eliceiri, K.-W., 2012, NIH Image to ImageJ: 25 years of image analysis, Nature methods, 9/7: 671-675.

[19] Zhang, H.-X., Du, G.-H., Zhang, J.-T., 2004, Assay of mitochondrial functions by resazurin in vitro, Acta pharmacologica Sinica, 25/3: 385-389.

[20] Wang, W., Caetano, G.-F., Chiang, W.-H., Braz, A.-L., Blaker, J.-J., Frade, M.-A., Bartolo, P.-J., 2017, Morphological, mechanical and biological assessment of PCL/pristine graphene scaffolds for bone regeneration, International Journal of Bioprinting. 2/2: 204-13.

[21] Goddard, J.-M., Hotchkiss, J.-H., 2007, Polymer surface modification for the attachment of bioactive compounds, Progress in polymer science, 32/7: 698-725.

[22] Wavhal, D.-S., Fisher, E.-R., 2002. Hydrophilic modification of polyethersulfone membranes by low temperature plasma-induced graft polymerization, Journal of Membrane Science, 209/1, 255-269. 
Click here to download high resolution image

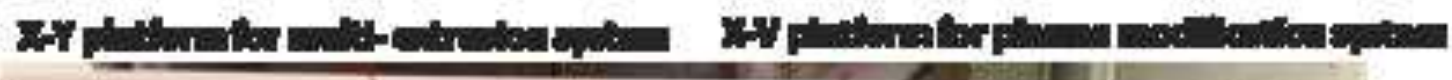

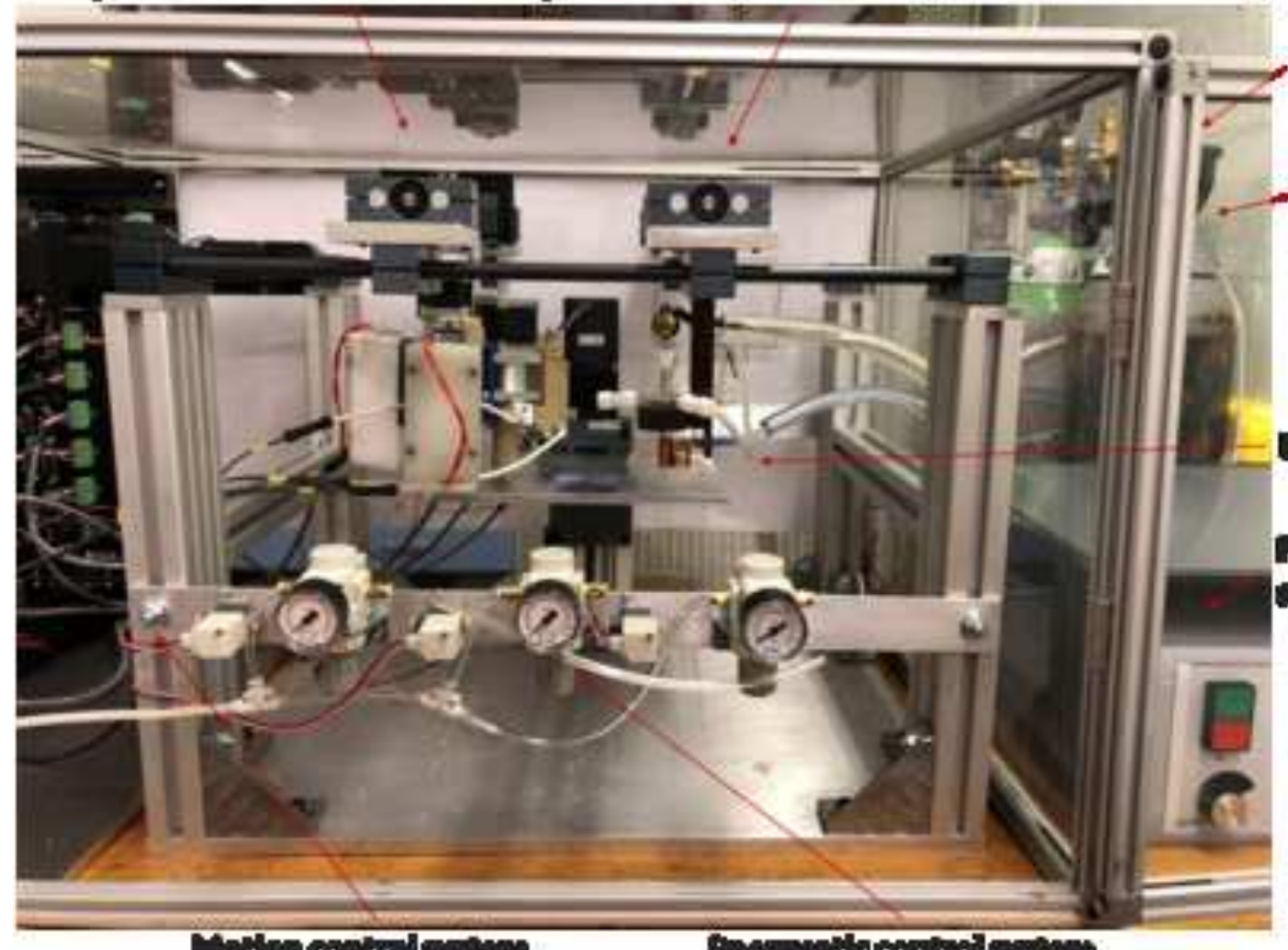

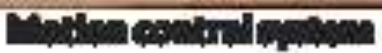

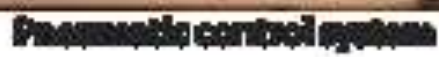

(a)

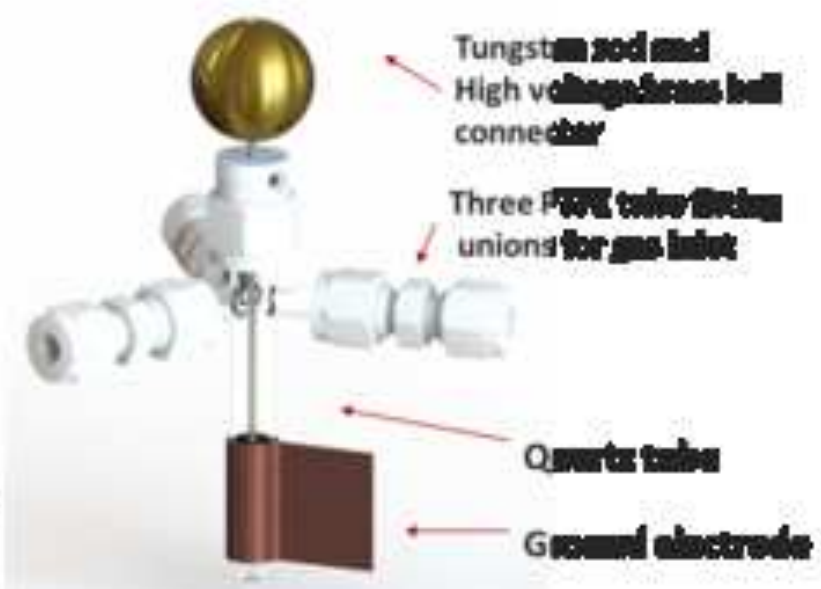

b)

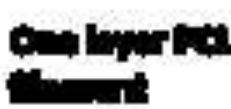

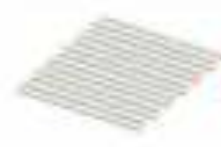

E.

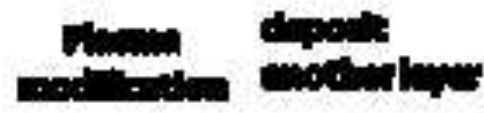

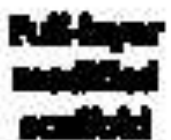

3 S

$\rightarrow 2$

다이

대에

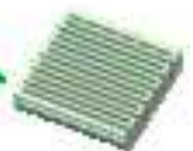

th 
Click here to download high resolution image

(a)

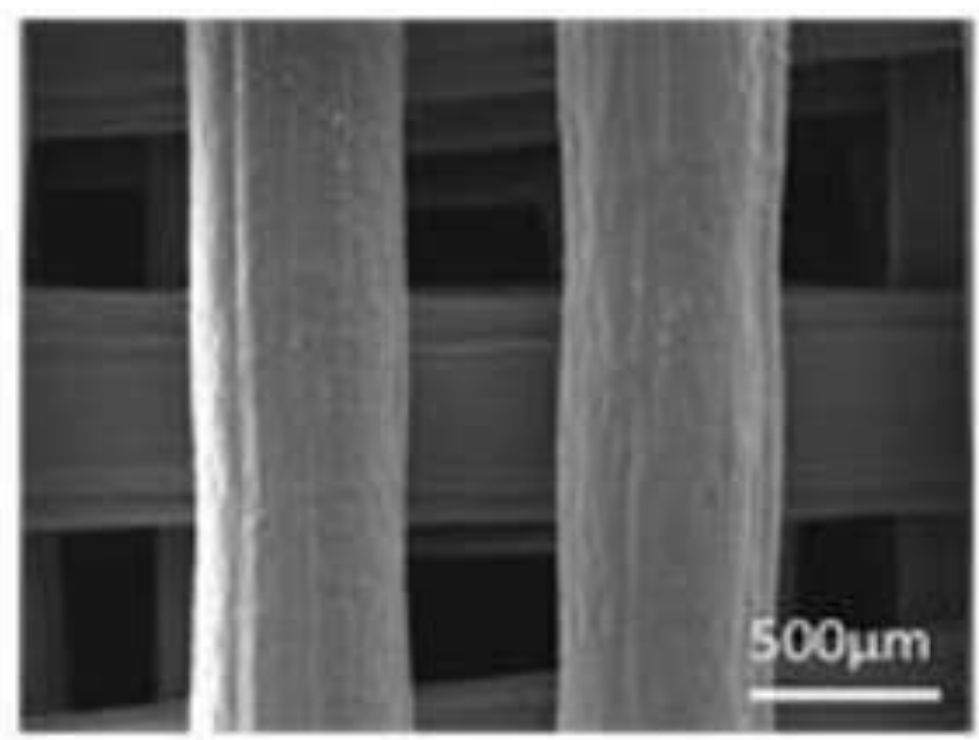

Top view

(b)

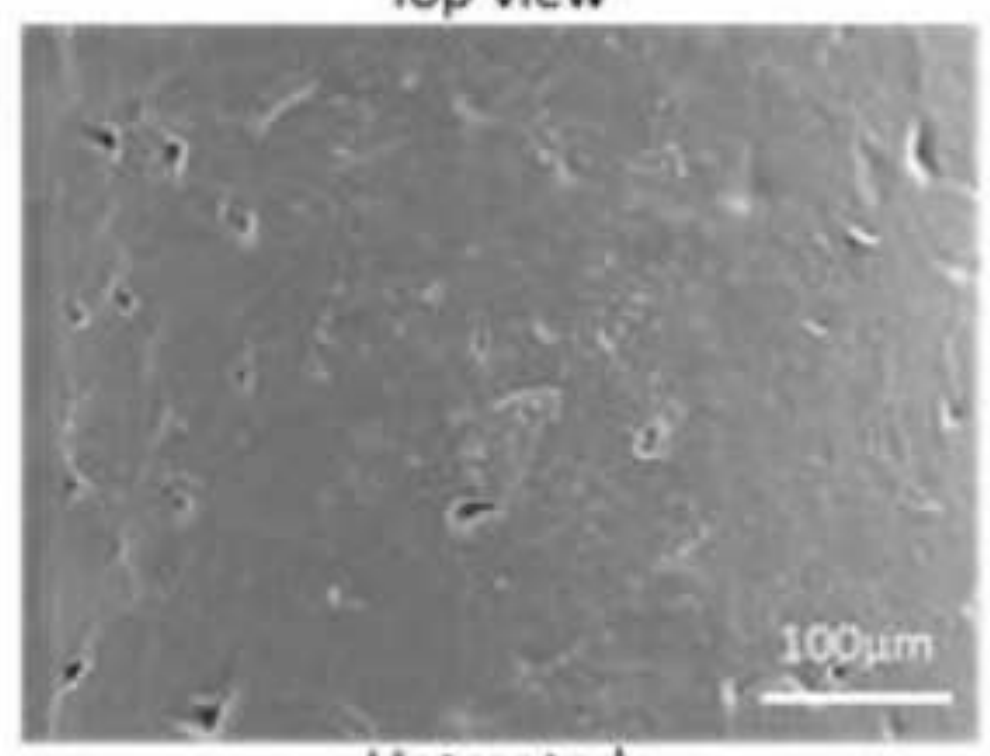

Untreated

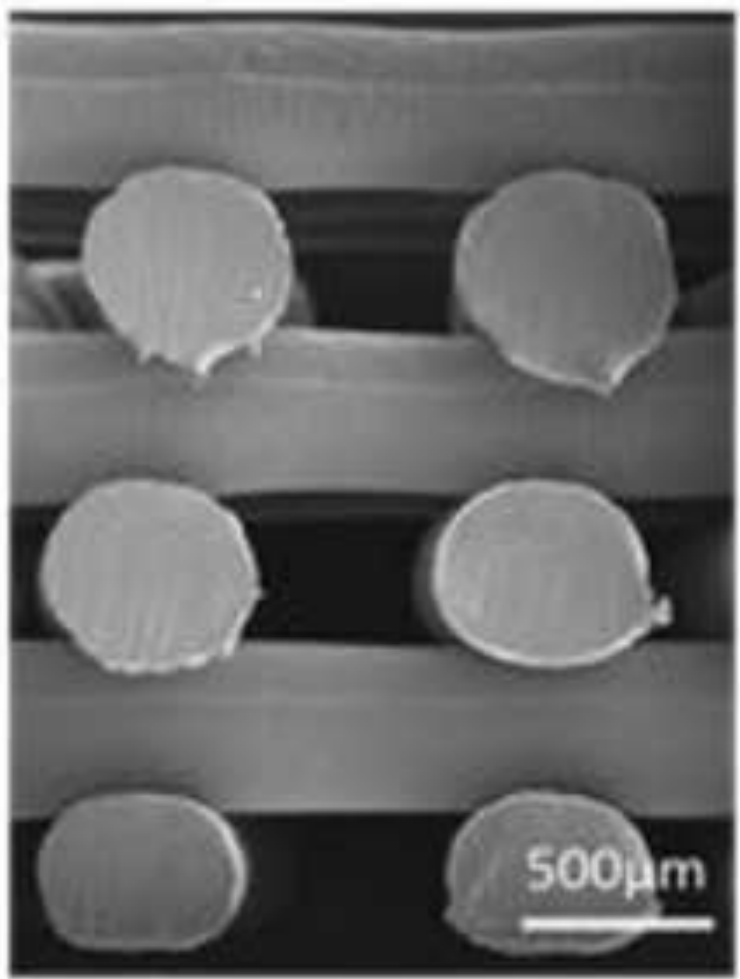

Side view

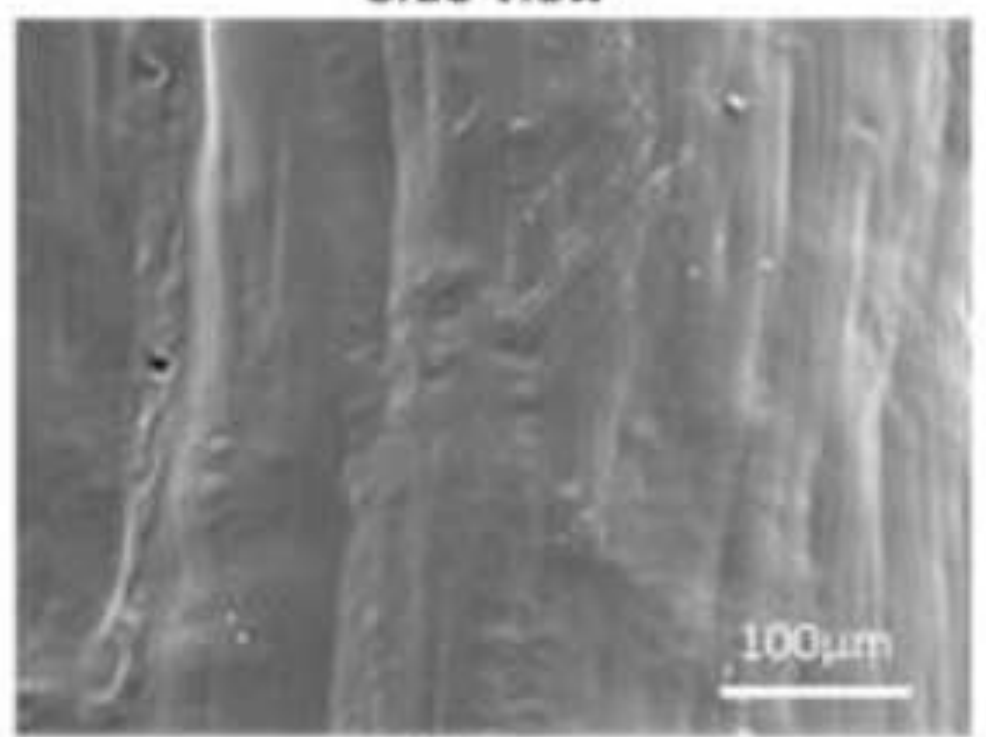

N2 plasma treated 


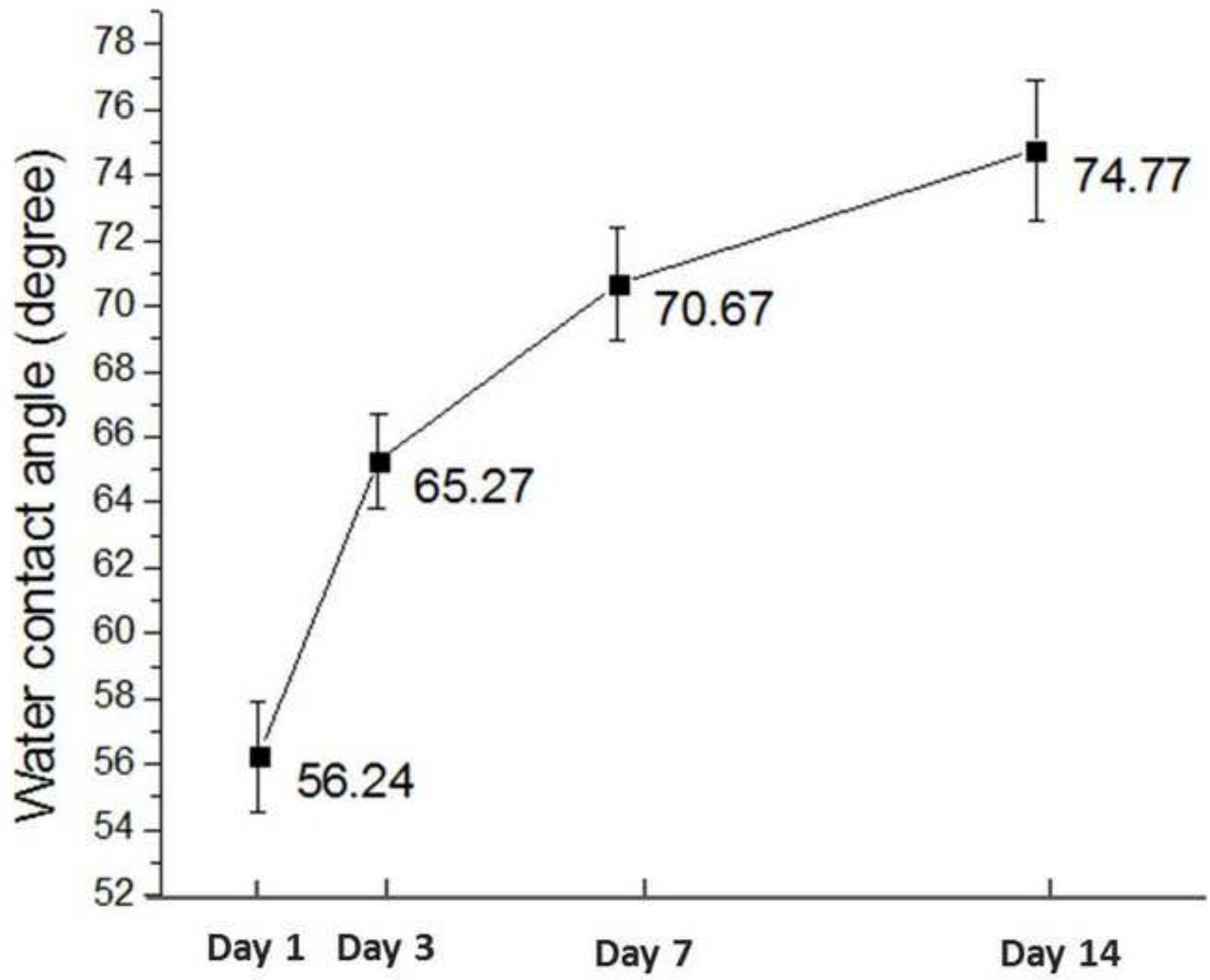




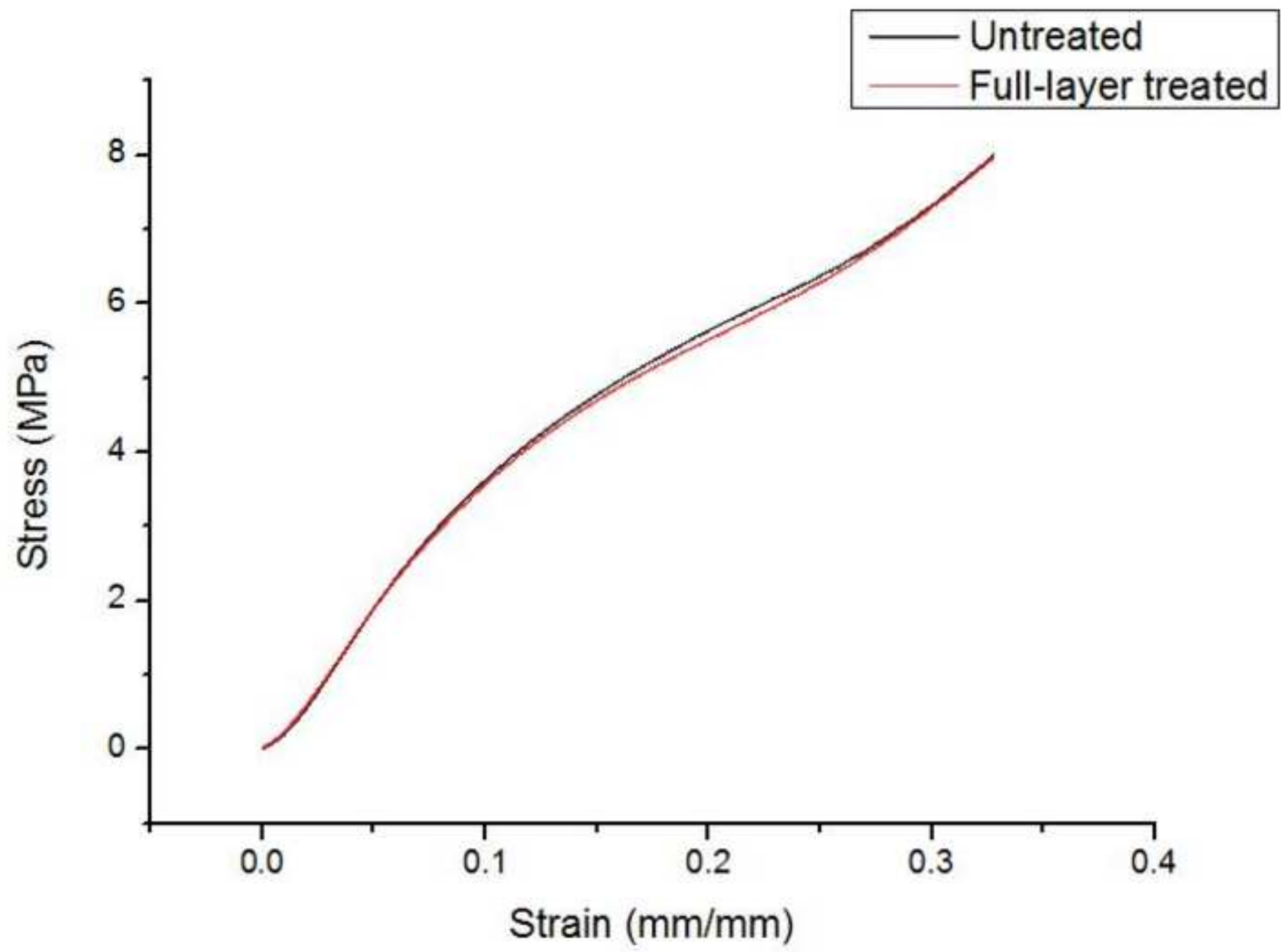




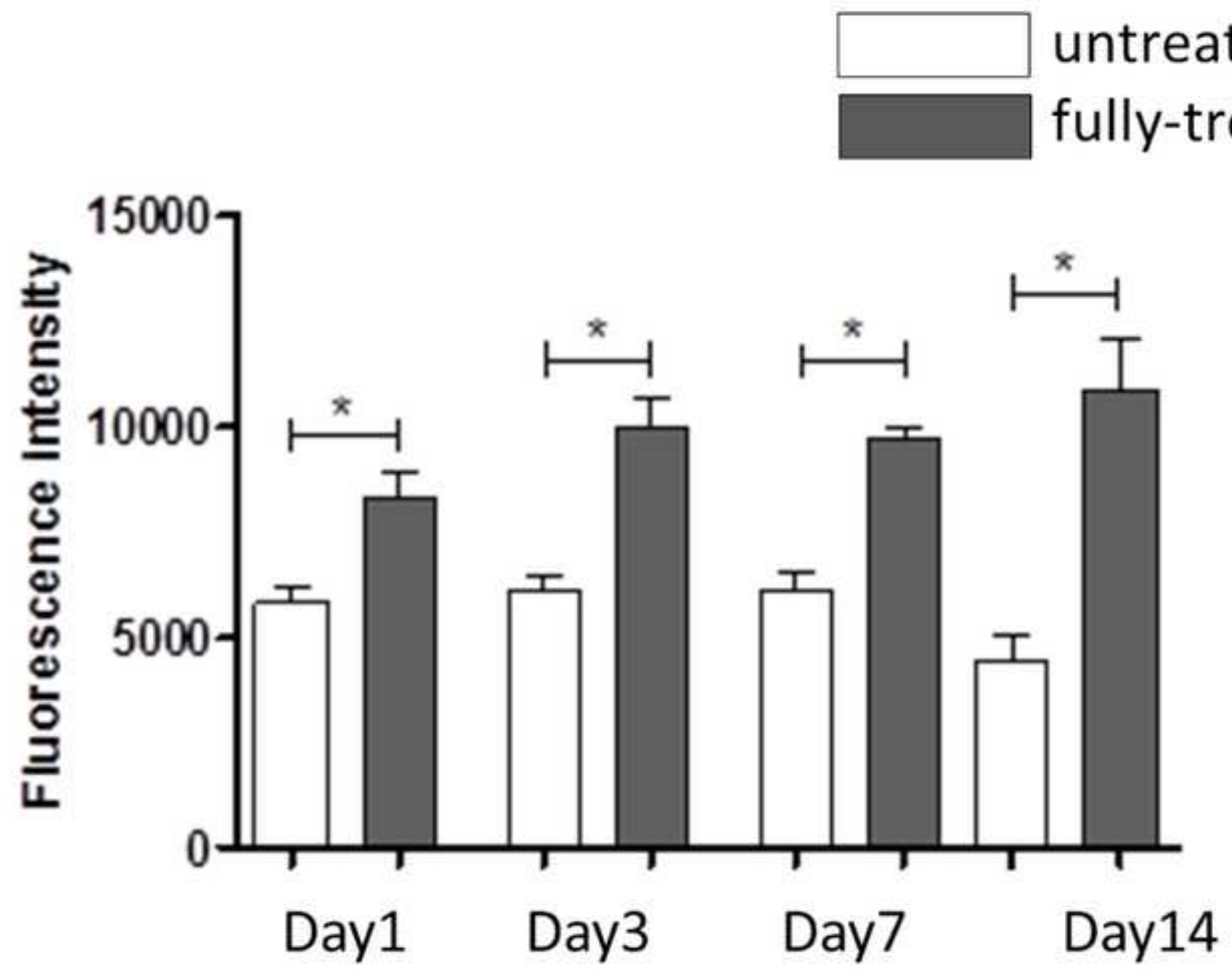


Table 1

Table 1 Temporal variation of water contact angles for treated and untreated scaffolds

\begin{tabular}{lll} 
& PCL scaffolds & $\mathrm{N}_{2}$ plasma fully treated \\
\hline $0 \mathrm{~s}$ & $83.2 \pm 2.0$ & $63.0 \pm 3.1$ \\
\hline $0.5 \mathrm{~s}$ & $82.9 \pm 1.2$ & $26.7 \pm 0.9$ \\
\hline $3 \mathrm{~s}$ & $80.9 \pm 2.7$ & Fully absorbed
\end{tabular}


1. The keywords in the manuscript are not from the list of CIRP keywords. Two keywords from the CIRP list have been used in the revised paper.

2. A relevant ref. to support the statement of tissue engineering and biomanufactuirng is obsolete.

Reference to a CIRP paper is made to support this statement. (see reference 1)

3. No CIRP paper references.

References to two Annals CIRP papers and one Procedia CIRP paper are now included.

4. Why would Biomanufacturing be constrained to AM techniques?

The sentence has now been revised.

5. Please put full name of PCL for the first use.

Done.

6. Figure $1(\mathrm{~b})$ is not clear. Please replace it with better image. A new version of Fig. 1(b) is used.

7. Figure 1 (c) gives no additional insights and shows normal control systems, so I suggest to remove it and instead increase the size of Figure 1 (a). Figure 1(c) deleted and the size of Fig. 1(a) increased.

8. In section 3.2, the plasma effect decrease with time. How can this be explained? Explanation inserted, including an additional reference.

9. In section 4(ii), the conclusion cannot be addressed from the compression test. Apologies. Mistake on our part. Now rectified.

10. In Figure 3, the $\mathrm{X}$ axis is uneven scaling. Figure 3 redrawn with a uniform $\mathrm{x}$-axis scale. The results now exhibit a non-linear behaviour.

11. In the text for the scaffold production, the authors say they used an extrusion speed of $15 \mathrm{rpm}$, however in their published paper [15], the author used a given speed of 12 rpm, why?

As we have in another publication (reference 17), higher screw rotational speed give rise to a better mechanical behaviour. Hence, the results presented in this paper were obtained at the higher rotational speed.

We have also replace the SEM images in Fig. 2(a) with a more recent image.

12. The diagram containing SD miss information about the number of experiments carried out.

The number of samples used for both mechanical (section 2.5) and biological tests (section 2.6) are mentioned.

13. The authors draw conclusions regarding mechanical properties from one experiment. The mechanical tests were repeated four times. This information is now included in section 2.5.

14. Figure 5 is of a bad quality and must be improved. Revised figure used.

15. Points after abbreviations of first names of the authors are missing Points inserted.

16. There is no clear indication of the affiliation.

Done. 
17. It is not a really a conclusion.

Conclusions revised. 